\title{
DERIVING THE AHP 1-9 SCALE FROM FIRST PRINCIPLES
}

\author{
Thomas L. Saaty \\ University of Pittsburgh \\ Pittsburgh, PA 15260 \\ saaty@katz.pitt.edu
}

Dedicated to my distinguished friend and colleague, Professor Dr. Klaus Dellmann

Keywords: eigenvector, eigenfunction, consistency, stability, homogeneity

Summary: We demonstrate how the integers 1 to 9 used in the Fundamental Scale of the AHP to represent pairwise comparison judgments can be derived from stimulus-response theory. The conditions required for the stability of the eigenvector of priorities, known from the mathematics literature, are briefly mentioned. These conditions require that the elements being compared be homogeneous. This limits the upper value of the scale to 9. They also require that the number of elements compared be small. It is widely known that both of these conditions are intrinsic to the way in which our brains actually operate. A brief discussion is given about two ways to deal with a large number of elements, both included in the AHP protocol.

\section{Introduction}

Paired comparisons are a natural process that people are able to do in expressing their sense of preference, importance or likelihood with respect to a certain property that the elements being compared have in common. When paired comparisons are made on the conscious level, they involve thought and reflection and therefore are discrete in time. But they also occur at the subconscious to perform decisions about the body: is sleep more important than eating late at night, is the body warm enough as compared with the outside temperature to go for a walk. Signals received from the body are continuously processed according to their urgency or priority. Thus, continuous paired comparisons are also part of our natural endowment. It is widely believed that the mechanisms of our conscious behavior derive from and are outward manifestations closely linked to the subconscious.

We show here how the process of deriving a priority eigenvector that represents ratio relations among the elements of a discrete decision represented by a positive reciprocal matrix of paired comparisons based on ratios, can be extended to the continuous case to derive an eigenfunction of priorities as the solution of a functional equation also based on ratios.

From such an eigenfunction we obtain the well known Weber-Fechner law of response to stimuli as a first order approximation. We then use this response function to derive expressions for response to just noticeable successive stimuli. The ratios of these successive responses lead to the integer valued scale of the Analytic Hierarchy Process for decision-making (AHP) [2]. By examining the sensitivity of the derived principal eigenvector to perturbations in the scale we find that the number of elements in the comparisons must be small and the values assigned must be homogeneous (of the same order of magnitude) from which we conclude that the values which begin with 1 must not go past the value 9 . We also show that the upper limit of 9 does not impose a severe limit on comparisons of widely disparate elements by using clustering with a common pivot from one cluster to the next carefully constructed so the scale values of a cluster differ by just one order of magnitude from an immediately adjacent cluster. 


\section{Deriving the Integer Valued Scale}

Paired comparisons in the AHP are given in terms of consistent and near consistent matrices. The latter are small perturbations of the former. The consistent set up leads to computing the principal eigenvector of the following equation written out in slightly elaborated but familiar matrix form:

$$
A w=\begin{array}{ccr}
A_{l} & \cdots & A_{n} \\
A_{l} & {\left[\begin{array}{rrr}
\frac{w_{l}}{w_{l}} & \cdots & \frac{w_{l}}{w_{n}} \\
\vdots & & \vdots \\
A_{n} & & \\
\frac{w_{n}}{w_{l}} & \cdots & \frac{w_{n}}{w_{n}}
\end{array}\right]\left[\begin{array}{r}
w_{l} \\
\vdots \\
w_{n}
\end{array}\right]=n\left[\begin{array}{r}
w_{l} \\
\vdots \\
w_{n}
\end{array}\right]=n w .}
\end{array}
$$

When written out as a system of equations we have

$$
\sum_{j=1}^{n} a_{i j} w_{j}=\lambda_{\max } w_{i} \quad \mathrm{i}=1, \ldots, \mathrm{n}
$$

subject to: $a_{j i}=1 / a_{i j}$ (or simply $a_{i j} a_{j i}=1$ ) known as the reciprocal condition resulting from the stronger consistency condition $a_{i j} a_{j k}=a_{i k}, i, j, k=1, \ldots, n$, and the normalization condition $\sum_{i=1}^{n} w_{i}=1$.

In continuous form our paired comparison process replaces the matrix with a kernel function $K(\mathrm{~s}, \mathrm{t})$ of an integral operator, the summation sign by an integral and $w_{j}$ and $w_{i}$ by $w(t)$ and $w(s)$ respectively. Here as in the matrix $A$ above, the kernel satisfies the reciprocal condition $K(s, t) K(t, s)=1$ that is also a result of consistency defined by

$$
K(s, t) K(t, u)=K(s, u) \text {, for all } s, t \text { and } u \text {. }
$$

Our problem of extracting the principal eigenvector takes the familiar form of Fredholm's equation of the second kind

$$
\int_{a}^{b} K(s, t) w(t) d t=\lambda_{\max } w(s)
$$

classically treated in the form

$$
\lambda \int_{a}^{b} K(s, t) w(t) d t=w(s)
$$

with the normalization condition $\int_{a}^{b} w(s) d s=1$.

It is easy to show that a consistent kernel has the form $K(s, t)=k(s) / k(t)$ from which what I call the "response" eigenfunction w(s) solution can be shown to be

$$
w(s)=\frac{k(s)}{\int_{s} k(s) d s}
$$

Since the denominator is a constant, we can write $w(s)=\alpha k(s)$.

Generalizing on the discrete approach in which the consistent matrix A has rank one, we assume that the kernel $K(s, t)$ is homogeneous of order 1 . Thus, we have: 


$$
K(a s, a t)=a K(s, t)=k(a s) / k(a t)=a[k(s) / k(t)] .
$$

It follows that $w(a s)=\alpha k(a s)=\alpha a k(s)=\beta k(s)=\gamma w(s)$. This equation simplifies to the functional equation in the eigenfunction $w(s)$ as $w(a s)=b w(s)$.

The solution of this functional equation is also the solution of Fredholm's equation and is given by the general damped periodic response eigenfunction $w(s)$ to a stimulus of magnitude $s$ as follows [4]:

where $P$ is periodic of period 1 and $P(0)=1$.

$$
w(s)=C e^{\log b \frac{\log s}{\log a}} P\left(\frac{\log s}{\log a}\right)
$$

The well-known Weber-Fechner logarithmic law of response to stimuli can be obtained as a first order approximation to our eigenfunction through series expansions of the exponential and of the cosine functions $(\cos \mathrm{u} / 2 \mathrm{~B})$ for which $P(0)=1$ as:

$$
v(u)=C_{1} e^{-\beta u} P(u) \approx C_{2} \log s+C_{3}
$$

where $P(u)$ is periodic of period $1, u=\log s / \log a$ and $\log a b \equiv-\beta, \beta>0$.

The expression on the right is known as the Weber-Fechner law of logarithmic response $M=a \log s+b, a \neq 0$ to a stimulus of magnitude $s[1,3]$.

The integer-valued scale of response in the form of paired comparison judgments in the AHP can be derived from this the logarithmic response function in the following way:

For a given value of the stimulus, the magnitude of response remains the same until the value of the stimulus is increased sufficiently large in proportion to the value of the stimulus, thus preserving the proportionality of relative increase in stimulus for it to be detectable for a new response. This suggests the idea of just noticeable differences (jnd), well-known in psychology. Thus, starting with a stimulus $s_{0}$ successive magnitudes of the new stimuli take the form:

$$
\begin{gathered}
s_{1}=s_{0}+\Delta s_{0}=s_{0}+\frac{\Delta s_{0}}{s_{0}} s_{0}=s_{0}(1+r) \\
s_{2}=s_{1}+\Delta s_{1}=s_{1}(1+r)=s_{0}(1+r)^{2} \equiv s_{0} \alpha^{2} \\
s_{n}=s_{n-1} \alpha=s_{0} \alpha^{n}(n=0,1,2, \ldots)
\end{gathered}
$$

We consider the responses to these stimuli to be measured on a ratio scale $(b=0)$. A typical response has the form $M_{i}=a \log \alpha^{i}, i=1, \ldots, n$, or one after another they have the form:

$$
M_{1}=a \log \alpha, M_{2}=2 a \log \alpha, \ldots, M_{n}=n a \log \alpha
$$

We take the ratios $M_{i} / M_{1}, \mathrm{i}=1, \ldots, \mathrm{n}$ of these responses in which the first is the smallest and serves as the unit of comparison, thus obtaining the integer values $1,2, \ldots, \mathrm{n}$ of the fundamental scale of the AHP. It appears that numbers are intrinsic to our ability to make comparisons, and were not invented by our primitive ancestors. We must be grateful to them for the discovery of the symbolism.

Why the upper limit of the scale is 9 is due to the requirement of homogeneity (see section 4) as a condition for the stability of the eigenvector of priorities that also depends on comparing a few elements at a time. What does one do when this number of alternatives is large? The first is that the AHP has another mode for rating elements one at a time known as absolute measurement. It requires creating 
intensity scales for the covering criteria that are then used to rate the alternatives one at a time. The other is to use paired comparisons by clustering the elements in the comparisons as we now show.

\section{No Limit on the Range of the Scale: from 1-9 to 1- $\infty$}

We can use clustering as in the following example to compare a small cherry tomato with a large watermelon to show that all we need is the 1-9 scale to compare homogeneous elements and link them to other such elements successively by taking the largest element in a cluster and using it as the smallest one in the immediately next cluster. We divide all the priorities in a cluster by the priority of the pivot in that cluster, and multiply by its priority in the previous cluster. In this way, all the priorities of the clusters can be combined.

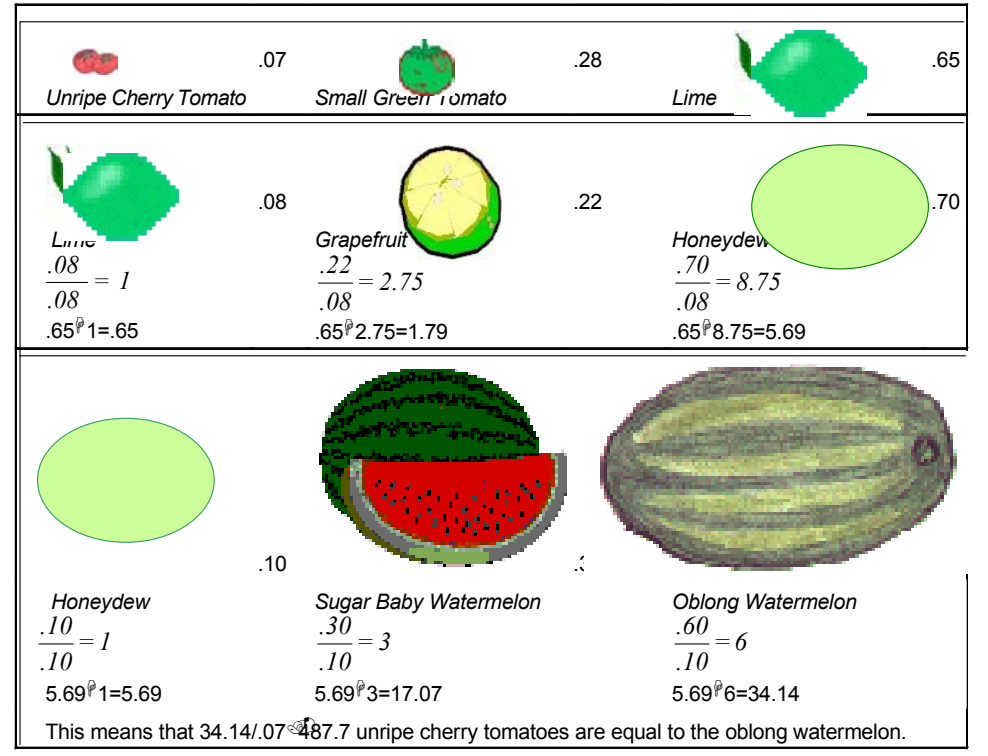

\section{On the Stability of the Eigenvector - the Need for Homogeneity}

Perturbing the matrix by adding to it the perturbation matrix $\Delta A$ yields the following perturbation $\Delta w_{1}$ in the principal eigenvector $w_{l}$. The following expression, due to J.H. Wilkinson [5], involves all the eigenvalues $8_{\mathrm{j}}$ of $A$ and all of both its left $\left(v_{j}\right)$ and right $\left(w_{j}\right)$ eigenvectors.

$$
\Delta w_{1}=\sum_{j=2}^{n}\left(v_{j}^{T} \Delta A w_{1} /\left(\lambda_{1}-\lambda_{j}\right) v_{j}^{T} w_{j}\right) w_{j}
$$

Left and right eigenvectors are in normalized form.

The eigenvector $w_{1}$ is stable when:

1) The perturbation $\Delta A$ is small as observing the consistency index would ensure;

2) $\lambda_{j}$ is well separated from $\lambda_{1}$; when $A$ is consistent, $\lambda_{1}=n, \lambda_{j}=0$;

3) The product of left and right eigenvectors is not too large which is the case for a consistent (and nearconsistent) matrix if the elements are homogenous with respect to the criterion of comparison and if their number is small. 


\section{References}

1. Batschelet, E., Introduction to Mathematics for Life Scientists, Springer-Verlag, New York, 1973.

2. Saaty, T.L., A Scaling Method for Priorities in Hierarchical Structures, J. Math. Psychology, vol. 15, no. 3, pp. 234-281, June 1977b.

3. — The Analytic Hierarchy Process, McGraw-Hill, New York, 1980; and RWS Publications, 4922 Ellsworth Avenue, Pittsburgh, PA, 1996.

4. —, The Brain: Unraveling the Mystery of How It Works, RWS Publications, 4922 Ellsworth Avenue, Pittsburgh, PA 15213, 2000.

5. Wilkinson, J.H., The Algebraic Eigenvalue Problem, Clarendon Press, Oxford, 1965. 
